\title{
A Clinical Report on Babesiosis as one of the Etiological Factors of Convulsions and Neurological Symptoms in Dogs and Cats in Tabriz With Emphasis on the Importance of Babesiosis in Public Health
}

\author{
Ramin Kaffashi Elahi ${ }^{*}$ (D) \\ ${ }^{1}$ Department of Clinical Sciences, Faculty of Veterinary Medicine, Tabriz Branch, Islamic Azad University, Tabriz, Iran
}

\begin{abstract}
Introduction: Babesiosis is a disease caused by protozoan parasites of the genus Babesia. It is transmitted by ticks and affects a wide range of domestic and wild animals and even humans.

Case Presentation: In this study, during the last three and a half years (2017-2021), 18 cats and 8 dogs with Babesia infection have referred to one of the small animal clinics in Tabriz, Iran. Ten of these 18 cats presented with obvious neurological symptoms, of which 7 had severe ocular symptoms in addition to neurological symptoms. The remaining eight had anemia and ocular symptoms and no neurological symptoms. In addition to ocular symptoms, one of the cases showed jaundice and anemia. All cases were in a critical condition at the time of referral to the clinic; that is, they showed all the expected symptoms and were close to death.

Conclusion: The aim of this article is to show Babesia as one of the etiological factors of convulsions and neurological symptoms in dogs and cats

Keywords: Clinical report, Babesiosis, Dog and cat, Neurological symptoms, Tabriz
\end{abstract}

Received: November 12, 2020, Accepted: December 15, 2020, ePublished: January 1, 2021

\section{Introduction}

Babesiosis is a disease caused by protozoan parasites of the genus Babesia. Babesiosis is transmitted by ticks and affects a wide range of domestic and wild animals and even humans (1). Ticks are the main vectors of Babesia. This is because Babesia protozoa spend most of their lives in the ticks. Ticks are therefore the main source of disease transmission. Note that several generations of these protozoa may remain in the ticks. This causes the disease to be transmitted to animals. The main complications of this disease include enlarged and very fragile spleen, swollen liver, enlarged gallbladder, compressed and black colored Kidneys, anemia, and jaundice (2). Additionally, brown or red urine due to hemoglobinuria was observed in most cases of Babesia infection. Disruption of blood coagulation and blockage in other organs, including the brain and heart, are damages caused by this disease. Babesiosis is diagnosed based on clinical signs; however, in some cases, it can be confused with other diseases that cause fever, jaundice, anemia, hemolysis, and redness of the urine. Therefore, for a definitive diagnosis of this disease, detailed and microscopic examinations of the blood should be performed. Thick, thin specimens should preferably be obtained from the capillaries in the ear or tail of a live animal (3). If the animal dies, it should be necropsied and the muscles of the heart, kidneys, liver, lungs, brain, and blood vessels should be examined. Babesia species can be identified morphologically by light Microscope.

Several serological tests are performed to detect antibodies to Babesia in animals, the most common of which are indirect fluorescent antibody testing and enzyme-linked immunosorbent assay (ELISA). PCR tests are also able to detect very low parasitemia (4).

Several species of Babesia, including B. duncani, $B$. venatorum, $B$. conradae, can cause babesiosis in humans.

Babesia infections in humans are caused by the bite of infected ticks or by receiving infected blood. The disease has been reported to be extremely dangerous and even fatal in people who have undergone splenectomy or in people with severe immunodeficiency (5).

Dogs are more likely to be infected with Babesia than 
cats. The most important species of Babesia that cause disease in dogs and cats are B. canis, B. vogeli, and B. rossi.

One of the most important causes of disease in dogs is B. gibsoni, which is very small in size. This parasite can cause chronic disease with severe and progressive anemia (6).

Babesia felis has also been reported frequently in domestic cats in South Africa (7).

\section{Case Presentation}

In this study, during the last three and a half years (20172021), 18 cats and 8 dogs with Babesia infection have referred to one of the small animal clinics in Tabriz, Iran. Ten of these 18 cats presented with obvious neurological symptoms, of which 7 had severe ocular symptoms in addition to neurological symptoms (Figure 1). The remaining eight had anemia and ocular symptoms and no neurological symptoms.

In addition to ocular symptoms, one of the cases showed signs of jaundice and anemia. All cases were in a critical situation at the time of referral to the clinic; in other words, they showed all the expected symptoms and were close to death. None of the dogs had ocular symptoms. All dogs showed signs of jaundice and anemia. None of the dogs had peripheral lymphadenopathy. Paraclinical tests also showed that half of them had elevated liver enzymes. All cases showed signs of improvement after the first dose of anti-Babesia drugs, and all were apparently healthy within two weeks. Consecutive blood tests at three-week intervals showed that blood parameters returned to normal by the second month after treatment, and three more subcutaneous injections were given every 14 days to completely clear the blood of the protozoan.

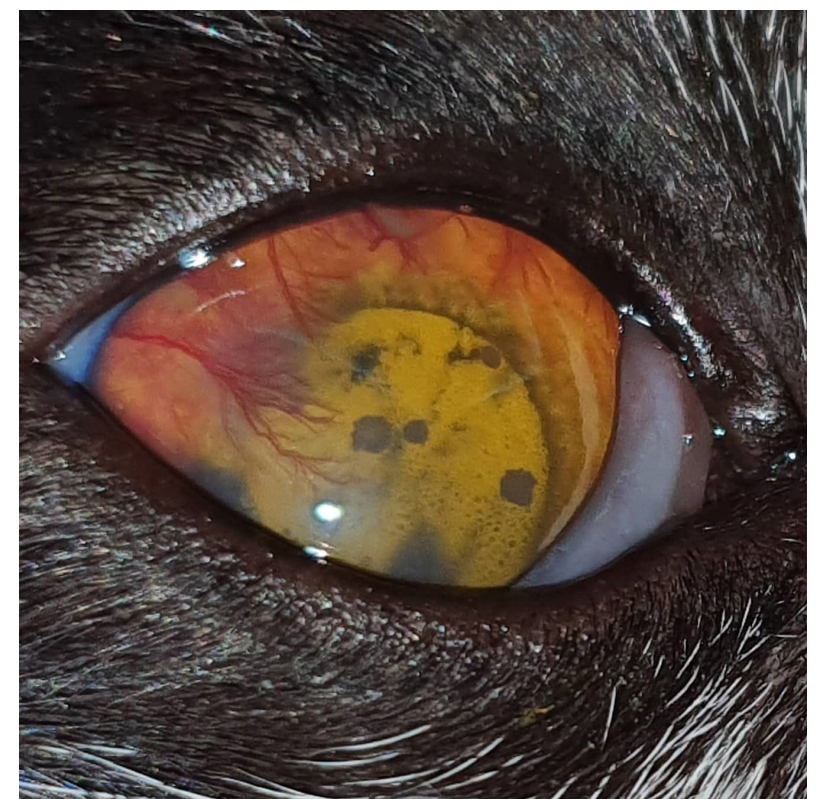

Figure 1. Eye of the Cat With Babesiosis With Symptoms of Congestions and Corneal Opacity.

\section{Discussion}

In this study, the cases were mostly cats over one year of age with predominantly neurological symptoms and convulsions that did not respond to anticonvulsant therapy. In some cases, peripheral lymphadenopathy was observed but they were normal in terms of other symptoms. Some cases showed only symptoms of anemia and hepatitis. Some cases showed only symptoms of anemia, but the important thing is that some cases had no symptoms other than convulsions and were normal in other respects, which is an important clinical challenge leading to clinical and diagnostic suspicions of a brain complication. Therefore, the importance of this article is that Babesia is suggested as one of the etiological factors of convulsions in suspected cases. For many years, patients with babesiosis referred with symptoms of anemia, mucosal pallor, and weight loss.

It seems necessary for veterinarians to consider the prevalence of ticks in contaminated areas and areas where cats are kept in unsanitary conditions. These recommendations along with the available clinical signs allow veterinarians to diagnose or suspect Babesia felis.

It is natural that the final diagnosis and confirmation will be made by blood cytological tests (8).

\section{Conclusion}

The aim of this study was to show Babesia as one of the etiological factors of convulsions and neurological symptoms in dogs and cats referred to animal clinics.

\section{Conflict of Interests}

The authors declared that no competing interests exist.

\section{Ethical Issues}

In this research, ethical considerations have been fully observed.

\section{Acknowledgements}

The author would like to express his deep gratitude to Islamic Azad University, Tabriz Branch, for offering valuable practical assistance to the research team in the present study.

\section{Funding}

The author received no financial support for the research, authorship, and publication of this article.

\section{References}

1. Julis P, Kreier JP. Parasitic Protozoa. 5th ed. New York: Academic Press Inc.; 1993. p. 1-34.

2. Greene CE. Infectious Diseases of the Dog and Cat. 4th ed. Elsevier Health Sciences; 2012.

3. Akhtardanesh B, Ghanbarpour R, Blourizadeh $\mathrm{H}$. Serological evidence of canine monocytic ehrlichiosis in Iran. Comp Clin Pathol. 2010;19(5):469-74. doi: 10.1007/ s00580-009-0889-5.

4. Anderson JF, Mintz ED, Gadbaw JJ, Magnarelli LA. Babesia microti, human babesiosis, and Borrelia burgdorferi in Connecticut. J Clin Microbiol. 1991;29(12):2779-83. doi: 10.1128/jcm.29.12.2779-2783.1991. 
5. Jefferies R, Ryan UM, Muhlnickel CJ, Irwin PJ. Two species of canine Babesia in Australia: detection and characterization by PCR. J Parasitol. 2003;89(2):409-12. doi: 10.1645/0022-3395(2003)089[0409:tsocbi]2.0.co;2.

6. Shaw SE, Day MJ. Arthropod-Borne Infectious Diseases of the Dog and Cat. 1st ed. United Kingdom: Mansons Press; 2005. p. 63-7.
7. Bashir IN, Chaudhry ZI, Ahmed S, Saeed MA. Epidemiological and vector identification studies on canine babesiosis. Pak Vet J. 2009;29(2):51-4.

8. Adaszek $\ell$, Martinez AC, Winiarczyk S. The factors affecting the distribution of babesiosis in dogs in Poland. Vet Parasitol. 2011;181(2-4):160-5. doi: 10.1016/j. vetpar.2011.03.059.

(C) 2021 The Author(s); This is an open-access article distributed under the terms of the Creative Commons Attribution License (http:// creativecommons.org/licenses/by/4.0), which permits unrestricted use, distribution, and reproduction in any medium, provided the original work is properly cited. 